\title{
edoc
}

Institutional Repository of the University of Basel

University Library

Schoenbeinstrasse 18-20

CH-4056 Basel, Switzerland

http://edoc.unibas.ch/

Year: 2006

\section{Verhaltensmedizin, Psychotherapie und Zahnheilkunde}

\author{
Gunther Meinlschmidt, Olivia Bolt
}

Posted at edoc, University of Basel

Official URL: http://edoc.unibas.ch/dok/A5255021

Originally published as:

Meinlschmidt, G. \& Bolt, O. (2006). Verhaltensmedizin, Psychotherapie und Zahnheilkunde. Verhaltenstherapie, 16, 2, 122-132.

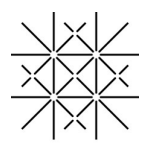




\section{Verhaltensmedizin, Psychotherapie und Zahnheilkunde}

Gunther Meinlschmidt ${ }^{a, b}$ Olivia Bolt ${ }^{a, b}$

${ }^{a}$ Abteilung für Klinische und Theoretische Psychobiologie, Universität Trier, Deutschland

${ }^{\mathrm{b}}$ Abteilung für Klinische Psychologie und Psychotherapie, Institut für Psychologie, Universität Basel, Schweiz

Dr. Gunther Meinlschmidt, Dipl.-Psych., Psychotherapeut

Abteilung für Klinische Psychologie und Psychotherapie

Institut für Psychologie, Universität Basel

Missionsstrasse 60/62, 4055 Basel, Schweiz

Tel. +41 6126702 75, Fax 2670278

E-mail gunther.meinlschmidt@unibas.ch 


\section{Schlüsselwörter}

Verhaltensmedizin · Verhaltenszahnmedizin · Oralpsychologie $\cdot$ Psychosomatik $\cdot$ Psychotherapie

\section{Zusammenfassung}

Einleitung: Bei der Entstehung und Behandlung zahnmedizinischer Störungsbilder sind biologische, psychologische und soziale Prozesse von Relevanz. Dennoch findet Wissen über das Zusammenspiel dieser Faktoren bisher nur selten Eingang in die klinische Praxis. Ziel des vorliegenden Beitrags ist es daher, einen Überblick über Berührungspunkte zwischen Verhaltensmedizin, Psychotherapie und Zahnheilkunde zu geben. Methoden: Die verhaltensmedizinischen Implikationen verschiedener zahnmedizinisch relevanter Erkrankungen und Störungsbilder (Zahnkaries, Gingivitis, Parodontitis, «Burning-mouth»-Syndrom und Mundgeruch) sowie zahnmedizinische Besonderheiten bei spezifischen Patientengruppen werden dargestellt. Ergebnisse: Das umfangreiche Wissen an der Schnittstelle von Verhaltensmedizin, Psychotherapie und Zahnheilkunde bietet vielfältige Ansatzmöglichkeiten für klinische Interventionen, deren Wirksamkeit zum Teil wissenschaftlich nachgewiesen ist. Diskussion: Die Integration von Wissen um psychobiologische Prozesse bei Psychotherapie einerseits und bei zahnmedizinischen Erkrankungen anderseits, kann Wege zu neuen, neuropsychotherapeutisch fundierten Interventionsmethoden aufzeigen. Es ist zu erwarten, dass weitere qualitativ hochwertige Studien zur Wirksamkeit einzelner Interventionen wichtige Beiträge zur Verbesserung zahnbezogener Gesundheit leisten können.

\section{Key Words}

Behavioral medicine $\cdot$ Behavioral dentistry $\cdot$ Oral psychology $\cdot$ Psychosomatics $\cdot$ Psychotherapy

\section{Summary}

Behavioral Medicine, Psychotherapy and Dentistry

Introduction: Biological, psychological and social processes are of relevance in the development and treatment of dental disorders. However, knowledge about interactions among these factors has only few implications for clinical practice. Hence, the goal of this article is to provide an overview of the links between behavioral medicine, psychotherapy and dentistry. Methods: The biobehavioral implications of different dental illnesses and disorders (caries, gingivitis, periodontitis, burning-mouth syndrome, and halitosis) as well as biobehavioral characteristics of specific patient groups are presented. Results: The existing scientific knowledge in the interdisciplinary field of behavioral medicine, psychotherapy and dentistry provides the basis for a variety of treatment approaches. Its efficacy has, in part, already been confirmed. Discussion: The integration of knowledge on psychobiological processes underlying psychotherapy and dental illnesses may lead to new interventions, based on the concept of neuropsychotherapy. It can be expected that further studies on the efficacy and effectiveness of specific biobehavioral interventions will significantly contribute to an increase in oral health. 


\section{Einleitung}

Intensivere Verbindungen zwischen Zahnmedizin und Verhaltenswissenschaften gehen bis in die 50er Jahre zurück [Cohen, 1981]. Die Zusammenarbeit der Disziplinen entwickelte sich jedoch zuerst sehr zögerlich. Heute liegt zu den Zusammenhängen psychosozialer Aspekte mit Zahnmedizin und Zahngesundheit ein breites Wissen vor. Im angloamerikanischen Raum hat sich dabei der Begriff «behavioral dentistry» (Verhaltenszahnmedizin) etabliert [Dworkin, 2000]. Im deutschsprachigen Raum wird der Bereich z.B. unter dem Ausdruck «Psychologie und Psychosomatik in der Zahnmedizin» oder seltener auch «Oralpsychologie» subsumiert. In Deutschland finden sich in verschiedenen Fachgesellschaften (Deutsche Gesellschaft für Medizinische Psychologie, Deutsches Kollegium für Psychosomatische Medizin, Deutsche Gesellschaft für Zahn-, Mund- und Kieferheilkunde) Arbeitskreise, die dieses Forschungs- und Anwendungsfeld vorantreiben und sich inzwischen unter dem Namen «PsyDent» (www.uni-duesseldorf.de/PsyDent) vernetzt haben. Im vorliegenden Artikel wird ein Überblick über die Berührungspunkte zwischen Zahnheilkunde, Psychotherapie und Verhaltensmedizin gegeben.

\section{Ausgewählte zahnmedizinische Fragestellungen}

\section{Zahnkaries}

Die gesunde Zahnhartsubstanz befindet sich in einem Gleichgewicht von Demineralisation und Remineralisation. Sie kann zerstört werden durch Milchsäure, die von dem Zahnschmelz anhaftenden Bakterien aus Kohlehydraten gebildet werden und zu einem kritischen Absinken des $\mathrm{pH}$-Wertes führen. Infolge dessen kommt es zu einer chemischen Veränderung und einem In-Lösung-Gehen der Zahnschmelzoberfläche. Der Zahnschmelz demineralisiert, so dass immer tiefere Bereiche des Zahnes, bis hin zum Zahnnerv angegriffen werden können. Dieser als Zahnkaries (Zahnfäule) bezeichnete Vorgang wird begünstigt durch mangelhafte Mundhygiene und die daraus entstehenden Plaques aus den Stoffwechselprodukten der Bakterien. Aber auch die Struktur des Zahnschmelzes, Zahnform und die Zusammensetzung des Speichels haben Einfluss auf die Entstehung von Zahnkaries. Zahnkaries ist eine der häufigsten Infektionskrankheiten. Mehr als 60\% aller Schulkinder und die meisten Erwachsenen in Industrieländern haben Karies [Petersen, 2003]. Glücklicherweise ist die Prävalenz von Zahnkaries in den letzten Jahren besonders bei Kindern und Jungendlichen deutlich zurückgegangen [Reich, 2001]. Die Hauptmaßnahmen der Kariesprophylaxe bestehen aus gründlicher Zahnreinigung, zahngesunder Ernährung und Zufuhr von Fluoriden. Daneben spielen regelmäßige Vorsorgeuntersuchungen eine wichtige Rolle.

Aus verhaltensmedizinischer Sicht steht bei Karies die Sicherstellung von adäquatem Zahnprophylaxeverhalten und der Inanspruchnahme zahnmedizinischer Versorgung im Vordergrund. Karies findet sich gehäuft bei Personen mit niedrigerem sozioökonomischem Status, wobei vermutlich Faktoren wie vermindertes Zähneputzen, geringere Verwendung fluoridierter Zahnpasta und geringere Inanspruchnahme zahnmedizinischer Versorgung eine vermittelnde Rolle spielen [Reisine und Psoter, 2001; Tickle et al., 1999, 2000]. Auch wenn Deinzer et al. [1998a, 2000] Zusammenhänge zwischen Stress und Speichelproteinen, die für die Entstehung von Karies bedeutsam sind, nachweisen gibt es im Gegensatz zu Parodontopathien nur wenige, ältere Studien, die einen Zusammenhang zwischen psychosozialem Stress und Karies feststellten [Sutton, 1965]. Andere Studien fanden keine direkte Verbindung zwischen Stress und Karies [Marcenes und Sheiham, 1992]. Deshalb ist eher nicht von einer generellen Notwendigkeit auszugehen, Interventionen zur Stressbewältigung bei Kariespatienten in Betracht zu ziehen, auch wenn dies im Einzelfall angebracht sein kann.

Verhaltensmedizinische Interventionen im Bereich Karies richten sich sowohl an die gefährdeten Personen selber als auch an deren Eltern oder Betreuungspersonen. Quinonez et al. [2001] konnten zeigen, dass Erziehungsbelastungen der Eltern das Auftreten von Zahnkaries vorhersagen. Daneben hängen Überzeugungen der Eltern zur Notwendigkeit regelmäßigen Zähneputzens mit der Zahnputzhäufigkeit der Kinder zusammen [Pine et al., 2000]. Kinder von Eltern mit einem stärkeren Kohärenzsinn, der als wichtiger salutogener Faktor gilt, besuchen, auch unabhängig von akuten Beschwerden, regelmäßiger den Zahnarzt und haben weniger Zahnkaries [Freire et al., 2002]. Verhaltensmedizinische Interventionsprogramme können sich diese Zusammenhänge zu Nutze machen, indem sie gezielt versuchen, die mit erhöhter Kariesgefahr der Kinder assoziierten Faktoren zu modifizieren. Weinstein et al., 2004 konnten in einer randomisierten Studie nachweisen, dass die gezielte Erhöhung der Motivation von Eltern zu zahnpräventiven Maßnahmen ihrer Kinder die Auftretenswahrscheinlichkeit von Zahnkaries bei den Kindern signifikant reduzierte.

\section{Parodontopathien}

Parodontopathien bezeichnen Erkrankungen des Zahnhalteapparats, die sich meist entzündlich, manchmal auch degenerativ, selten hyperplastisch darstellen.

Dazu zählen die gingivalen Erkrankungen, hauptsächlich die Gingivitis, bei der es zu einer oberflächlichen Entzündung des Zahnfleischsaumes kommt. Sie zeigt sich symptomatisch durch gerötetes und geschwollenes Zahnfleisch, das bei Kontakt (z.B. beim Zähneputzen oder bei der zahnärztlichen Untersuchung) spontan zu bluten beginnt, sowie durch Mundgeruch. Die 
Gingivitis hat weltweit bei Kindern wie auch bei Erwachsenen eine Prävalenz von 60-100\% [Cutress, 1986; Hugoson und Jordan, 1982].

Verschiedene Autoren berichten von einem Zusammenhang zwischen psychosozialem Stress und Zahnfleischsaumentzündungen [Deinzer et al., 1998; LeResche und Dworkin, 2002]. Es gibt Hinweise, dass stressbedingte mangelnde Mundhygiene und dadurch entstehende Plaque-Bildung einen vermittelnden Faktor zwischen Stress und Gingivitis darstellen [Deinzer et al., 2001]. Außerdem konnte gezeigt werden, dass unterschiedliche Formen von Stress mit einer veränderten Freisetzung sekretorischer Speichelproteine einhergehen, was zu einem veränderten Anhaften von Mikroorganismen an die Mundschleimhäute führen und damit die Anfälligkeit für Entzündungen im Mundbereich erhöhen kann [Bosch et al., 2003a, b]. Wird Gingivitis adäquat behandelt, klingt sie meist rasch ab. Unbehandelt besteht die Gefahr des Übergangs in eine Parodontitis (s.u.), so dass neben Stressbewältigungstraining und Etablierung adäquater Mundhygiene die Sicherstellung einer rechtzeitigen Behandlung die wichtigste verhaltensmedizinische Intervention bei Gingivitis darstellt. Neben der Gingivitis, stellt die Parodontitis - die Entzündung weiterer Teile des Zahnhalteapparates - die andere große Gruppe der Parodontopathien dar. Durch eine chronisch bakterielle Entzündung wird das Zahnfleisch und Stützgewebe (Parodont) angegriffen und zum Teil oder ganz zerstört, was zur Lockerung bis zum Verlust eines Zahnes führen kann. Neben der direkten Destruktion des Zahnhalteapparats durch die Erreger, kann auch das durch die Erreger aktivierte Immunsystem zur Zerstörung des Zahnhalteapparates beitragen [Breivik und Thrane, 2001].

Parodontitis tritt gehäuft auf in Folge schlechter Mundhygiene, supragingivalem Zahnstein, subgingivaler Konkremente, einer vorhergehenden Gingivitis, Rauchen, systemischen Erkrankungen (z.B. schlecht eingestellten Zuckerwerten bei Diabetes mellitus [Tsai et al., 2002]), Übergewicht [Al-Zahrani et al., 2003], genetischen Faktoren [Baker und Roopenian, 2002] und Stress [Al-Zahrani et al., 2003]. Ca. 45\% der Bevölkerung weltweit leiden an Parodontitis, 5-20\% unter schweren Formen [Petersen, 2003]. Als Folge können sich Infektionen in andere Körperbereiche ausbreiten. Außerdem zeigen Personen mit Parodontitis ein erhöhtes Risiko z.B. für Herzkreislauferkrankungen und Frühgeburten.

Zum Zusammenhang von Parodontitis, Belastungen und inadäquatem Umgang mit Stress liegen eine Vielzahl von Studien vor [Croucher et al., 1997; Genco et al., 1999; Genco et al., 1998; Hildebrand et al., 2000; Hugoson et al., 2002; Linden et al., 1996; Merchant et al., 2003; Monteiro da Silva et al., 1996]. Tierexperimentelle Studien konnten zeigen, dass unter anderem genetische Faktoren, lebensgeschichtlich frühe Belastungen sowie Veränderungen in Stresshormonen und diese regulierende Hirnstrukturen die Auftretenswahrscheinlichkeit von Parodontitis erhöhen [Breivik, Opstad et al., 2000; Breivik, Sluyter et al., 2000; Breivik, Stephan et al., 2002; Breivik, Thrane et al., 2002; Breivik et al., 2001; Shapira et al., 2000]. Interessanterweise kann jedoch ein durch solche Faktoren erhöhtes Risiko auch wieder durch gezielte physiologische oder behaviorale Interventionen reduziert werden [Breivik und Rook, 2000; Breivik, Thrane et al., 2000; Sluyter et al., 2002]. Beim Menschen gibt es Hinweise, dass eine lokale, jedoch nicht peripher erhöhte Konzentration verschiedener Zytokine bei chronischem und/oder akutem Stress gegebenenfalls verbunden mit gleichzeitiger oder auch späterer reduzierter Mundhygiene zu einer Verstärkung von Parodontopathien führt [Deinzer et al., 1999; Deinzer et al., 2004; Deinzer, Kottmann et al., 2000; Giannopoulou et al., 2003; Kamma et al., 2004; Mengel et al., 2002; Waschul et al., 2003].

Interessanterweise konnte kürzlich gezeigt werden, dass in der Entstehung von Parodontitis Interaktionen zwischen genetischen Prädispositionen und Verhalten auftreten. Allgemein führt Rauchen zu einer erhöhten Anfälligkeit für Parodontitis. Außerdem haben Menschen mit einem bestimmten Genotyp (eine bestimmte Ausprägung spezifischer Interleukin-1 assoziierter genetischer Polymorphismen) eine erhöhte Anfälligkeit für Parodontitis, allerdings nur wenn sie Raucher sind [Meisel et al., 2004; Meisel et al., 2002; Meisel et al., 2003]. Aus verhaltensmedizinischer Sicht ist dies von Relevanz, da dadurch Möglichkeiten entstehen, wie durch Verhaltensinterventionen (hier Raucherentwöhnung) die Auswirkungen eines potentiellen genetischen Risikos reduziert werden können. Es ist zu hoffen, dass in naher Zukunft Studien vorgelegt werden, die die Wirksamkeit verhaltensmedizinischer Interventionen bei Parodontalerkrankungen in randomisiertem und kontrolliertem Design untersuchen. In Abbildung 1 wird das Zusammenspiel wichtiger verhaltensmedizinisch relevanter Faktoren bei der Entstehung von Gingivitis und Parodontitis dargestellt.

\section{«Burning-mouth»-Syndrom}

Unter Mundbrennen ohne organische Ursachen («burning-mouth“-Syndrom, BMS) leiden besonders Frauen im mittleren bis höheren Lebensalter [Bergdahl und Bergdahl, 1999]. Berichtete Prävalenzzahlen schwanken je nach Definition und Studie zwischen 0,7 und 12,2\% [Bergdahl und Bergdahl, 1999; Hakeberg et al., 1997]. Besonders betroffen sind die vorderen 2/3 der Zunge, der harte Gaumen und die Lippen, seltener das Zahnfleisch, der Mundboden und der weiche Gaumen. Oftmals klagen BMS-Patienten zudem über Mundtrockenheit [Bergdahl und Bergdahl, 1999; Zakrzewska et al., 2001]. Veränderungen im zentralen Dopaminsystem bei Patienten mit BMS wurden berichtet [Hagelberg et al., 2003; Jaaskelainen et al., 2001]. Begleitet wird BMS oft von psychischen Störungen (z.B. Angstzuständen oder Depressionen) [Bogetto et al., 1998], Diabetes, Anämie und Mangelerscheinungen (Eisen, Folsäure, Vitamin-B-Komplex) sowie bestimmten oralen Dysfunktionen (Bruxismus, Zungenpressen). Außerdem fanden sich bei BMS Patienten vermehrt aversive Kindheitserfahrungen [Lamey et al., 2005]. Auch wenn verschiedene lokale, systemische und psychologische Faktoren mit BMS in Verbindung gebracht wurden, ist die Ätiologie von BMS noch weitgehend unverstanden.

Nach Ausschluss potentieller organischer Ursachen (wie bakterielle oder mykotische Infektionen, die meist medikamentös behandelt werden), erfolgt nach Diagnose von BMS die Therapie häufig symptomatisch, meist durch die Gabe eines 
Vitamin-B-Komplexes. Während die wissenschaftliche Fundierung der Gabe von Psychopharmaka angezweifelt wird, fand sich in einer Meta-Analyse von sieben Interventionsstudien, dass kognitive Verhaltenstherapie die Symptomstärke reduziert [List et al., 2003; Zakrzewska et al., 2003]. Für die Zukunft sind eine eindeutige Definition von BMS mit klaren Klassifikationskriterien, die Identifikation relevanter Subgruppen und weitere qualitativ hochwertige Studien zur Effektivität verschiedener Therapien und deren Kombinationen dringend von Nöten [Scala et al., 2003].

\section{Mundgeruch}

Halitose kann sowohl mundhöhlenbedingt (früher «Foetor ex ore“) als auch anderweitig verursacht sein (früher «Halitosis“ im engeren Sinne). Viele Personen mit Halitose leiden sozial oder psychisch stark unter dem Mundgeruch. Innerhalb des Mundbereiches sind die eigentliche Geruchsursachen fast immer Stoffwechselprodukte (VSC, volatile sulfur compounds) von bestimmten in versteckten Höhlungen oder dem hinteren Drittel des Zungenrückens liegenden gram-negativen Bakterien, die durch Abbau von Speiseresten, Blut, Zellen und einigen chemischen Komponenten entstehen und unangenehm riechen. Mundgeruch kann aber auch ausgehend von den oberen Atemwegen einschließlich der Nase (Stomatodysodia), zum Beispiel durch Mandel- und Kieferhöhlenentzündungen oder durch Pfeiffersches Drüsenfieber verursacht werden. Auch krankhafte Veränderungen in den unteren Luftwegen (z.B. Lungenabszesse und -tumore) können zu an Eitergeruch erinnernden Mundgeruch führen (Ozostomia). Als weitere Ursachen gelten pathogene Veränderungen des intestinalen Traktes, übel riechende Substanzen (z.B. Stoffwechselprodukte in Folge von Erkrankungen, wie Diabetes, Nierenleiden, Leber- oder Galleerkrankungen), einige Nahrungsmittel aber auch Medikamente, die vom Blutstrom in die Lungen gelangen und dort in die Atemluft übergehen [Tangerman, 2002].

Für verhaltensmedizinische Überlegungen ist die Unterscheidung zwischen genuiner Halitose, Pseudohalitose und Halitophobie von Relevanz. Unter genuiner Halitose versteht man wie oben dargestellt real vorhandenen Mundgeruch (als Folge von physiologischen oder pathologischen Bedingungen). Patienten mit Pseudohalitose (in schweren Fällen auch Halitophobie genannt) klagen unter Mundgeruch zu leiden, ohne dass dieser von Dritten feststellbar wäre [Murata et al., 2002]. Patienten mit Mundgeruchsbeschwerden zeigen vermehrt psychopathologische Symptome [Eli et al., 1996]. Zwischen 6-50\% aller Menschen klagen gelegentlichen über Mundgeruch, wobei bis zu 25\% stark darunter leiden [Bosy, 1997; Sanz et al., 2001]. Bei einer bedeutsamen Prozentzahl der Patienten, die medizinische Hilfe wegen Halitose aufsuchen, kann jedoch keine organische Ursache festgestellt werden [Ben-Aryeh et al., 1998]. Richter [1996] berichtet, dass nach einem standardisierten Therapieprotokoll zwar 99\% der Halitosepatienten keinen objektiv feststellbaren Mundgeruch mehr hatten, jedoch $24 \%$ der Patienten weiterhin überzeugt waren, dass ihr schlechter Atem zumindest zum Teil auch nach der Behandlung noch vorhanden war. Bei genuiner Halitose ist eine ursächliche Behandlung meistens gut möglich, zum Beispiel durch Beseitigung der zugrunde liegenden systemischen Erkrankung, Gebisssanierung oder Etablierung adäquater hygienischer Maßnahmen, besonders auch Zungenreinigung [Quirynen et al., 2002; Roldan et al., 2003]. Neben adäquaten diagnostischen und therapeutischen Fertigkeiten, sollten Zahnärzte gegebenenfalls auch schon in Risikogruppen (zum Beispiel bei Patienten mit Parodontitis) mögliche Beeinträchtigungen durch Mundgeruch thematisieren und adäquate Präventions- und Interventionsmöglichkeiten anbieten [Lenton et al., 2001]. Auch Patienten mit Pseudohalitose und Halitophobie wenden sich meist primär an eine zahnärztliche Praxis, so dass Zahnärzte in der Lage sein sollten, diese Gruppe von Hilfesuchenden zu erkennen und adäquat auf ihre Problematik zu reagieren. Die Selbstwahrnehmung von Mundgeruch wird aus verhaltensmedizinischer Sicht als ein multifaktorielles psychophysiologisches Geschehen begriffen, das eng mit dem eigenen Körperbild wie auch mit Persönlichkeitsaspekten zusammenhängt [Coil et al., 2002]. Bei Pseudohalitose oder Halitophobie, ist der Einbezug von verhaltensmedizinischen, beziehungsweise psychotherapeutischen Interventionen notwendig, die besonders bei schweren Fällen meist nicht von Zahnärzten geleistet werden können. Patienten mit schwerer Pseudohalitose schenken normalerweise dem Befund, dass kein Mundgeruch vorliegt, keinen Glauben. Neben der Vermittlung des Verständnisses, dass die Wahrnehmung des eigenen Atems, ähnlich wie die Wahrnehmung des eigenen Körpers, individuell höchst unterschiedlich ist, besteht bei milden Fällen die Möglichkeit zur Intervention, indem zum Beispiel Zahnärzte Geruchsproben sammeln, um die Geruchsbewertung zu objektivieren, objektive Messmethoden (Messgeräte) einsetzen, eine Vertrauensperson in die Diagnose, bzw. den Verlauf des vermeintlichen Mundgeruchs einbeziehen oder die Kontrollüberzeugung des Patienten bezüglich seiner Beschwerden erhöhen. Bei Bedarf sollte jedoch an Spezialisten (Verhaltensmediziner oder Psychotherapeuten) überwiesen werden, die dann mit verschiedenen Techniken die irrationalen Überzeugungen, Ängste und Interpretationen des Atemgeruchs sowie des sozialen Umfeldes modifizieren können, wobei gegebenenfalls weitere ätiologisch relevante Faktoren in die Therapie einbezogen werden müssen. Übermäßige (zahn)medizinische Maßnahmen können bei diesen Patienten zu einer Verstärkung der Überzeugung einer organischen Ursache führen und damit einer adäquaten Behandlung im Wege stehen und sind damit unbedingt zu vermeiden [Yaegaki und Coil, 2000]. Zurzeit beschränkt sich die Evidenz der Wirksamkeit verhaltensmedizinischer Interventionen bei Mundgeruch noch überwiegend auf Meinungen aus Expertenkreisen. Es ist zu hoffen, dass in naher Zukunft methodisch hochwertige, randomisierte und kontrollierte Studien die Wirksamkeit genauer in den Blick nehmen.

Die verschiedenen zahnmedizinische Krankheits- und Beschwerdebilder und dabei relevante verhaltensmedizinische Aspekte sind als Übersicht in Tabelle 1 dargestellt. 


\section{Spezifische Patientengruppen}

\section{Patienten mit psychischen Störungen}

Neben der Behandlung von psychischen oder Verhaltensaspekten bei Patienten mit zahnmedizinischen Problemen, stellen zahnmedizinische Probleme bei Patienten mit psychischen Störungen eine weitere wichtige Schnittstelle zwischen Psychologie und Zahnheilkunde dar.

Friedlander und Mahler [2001] machen darauf aufmerksam, dass Patienten mit Major Depression unter ausgeprägten zahnmedizinischen Beschwerden leiden können, und womöglich zahnmedizinische Behandlung aufsuchen noch bevor sie auf die psychische Problematik aufmerksam werden. Neben einer verstärkten Somatisierungsneigung können durch die Depression bedingte einseitige Ernährung, mangelnde Hygiene aber auch antidepressive Medikation zu den zahnmedizinischen Problemen führen. Auch andere mit der Depression einhergehende psychobiologische Veränderungen, z.B. des Endokrinums und des Immunsystems können womöglich Auswirkungen auf die Zahngesundheit haben. Wo immer möglich sollte eine zahnmedizinische Behandlung bei diesen Patienten nach deren Einverständnis in Rücksprache mit dem behandelnden Psychiater und/oder Psychotherapeuten erfolgen. Ähnliche Probleme bezüglich der Medikation können sich bei verschiedenen Angststörungen und anderen psychischen Erkrankungen ergeben [Friedlander et al., 2004]. Besondere Aufmerksamkeit sollte Patienten mit chronischem Erschöpfungssyndrom (chronic fatigue syndrom) geschenkt werden. Diese Patienten zeigen gehäuft temporomandibuläre Dysfunktionen und Schmerzbeschwerden. Auch die hier manchmal eingesetzten Medikamente können zu negativen zahnmedizinischen Zuständen führen. Bei der Terminierung der Behandlung muss gegebenenfalls auf die chronische Erschöpfungssymptomatik Rücksicht genommen werden, beziehungsweise bei komplexeren Eingriffen womöglich Rücksprache mit behandelnden Ärzten und/oder Psychotherapeuten genommen werden. Bei Patienten mit Essstörungen finden sich gehäuft zahnmedizinische Probleme. Die bei Anorexia nervosa (Magersucht mit und ohne Erbrechen) und Bulimia nervosa (Brechsucht ohne Untergewicht) oft vorliegenden Mangelzustände, wie auch die durch Erbrechen auftretenden Schäden durch Magensäure im Mundbereich (typisch sind Erosionen des Zahnschmelzes, besonders im Bereich der palatinalen Flächen der oberen Frontzähne) machen in vielen Fällen eine interdisziplinäre Behandlung unter Einbezug eines Zahnmediziners notwendig [Christensen, 2002; Gurenlian, 2002]. Bei Kindern mit Aufmerksamkeitsdefizithyperaktivitätsstörungen (ADHS) wurde ein um ein vielfaches erhöhtes Risiko für Zahnschäden festgestellt, was die dringende Notwendigkeit des Einbezugs zahnmedizinischer Überlegungen in die Behandlung dieser Kinder unterstreicht [Broadbent et al., 2004]. Außerdem gibt es erste Hinweise, dass es durch Medikation mit Psychostimulanzien bei Kindern mit ADHS womöglich zu einem vermehrten Auftreten von Bruxismus kommen kann [Malki et al., 2004].

Weitere sich auf die Zahngesundheit negativ auswirkende psychische Störungen sind zwanghafte Persönlichkeitsstörungen, Zwangsstörungen mit Zwangshandlungen oder Manien, bei denen exzessives Zähneputzen auftreten kann, Substanzmittelmissbrauch und psychotische Störungen, mit der Möglichkeit des Auftretens halluzinatorischer Körperempfindungen (Zönästhesien) im Mundbereich sowie mögliche negative Folgen der dabei eingesetzten Medikamente (z.B. verminderter Speichelfluss, die Mundhygiene beeinträchtigende Dyskinesien) [Longley und Doyle, 2003].

Die im Bezug zur Zahnmedizin wichtigsten psychischen Störungsgruppen sind in Tabelle 2 zusammengefasst.

\section{Kinder}

Besondere Bedeutung kommt der zahnärztlichen Behandlung von Kindern zu. Eine bereits im frühen Kindesalter etablierte, langfristig positive Einstellung gegenüber Zahnarztbesuchen ist Grundlage für nachhaltige Zahngesundheit.

Interventionen, die einen positiven Verlauf des Zahnarztbesuchs bei Kindern zum Ziel haben, beginnen bereits vor dem eigentlichen Zahnarztbesuch. Durch Kinderbücher, wie auch durch die Begleitung von älteren Kindern (Geschwistern) zu Zahnarztbesuchen, können Kinder mit der Situation und dem Ablauf in der Praxis in positiver Weise vertraut gemacht werden. Auch das Bereitstellen von Informationsmaterialien für die Eltern, mit der Aufforderung die Kinder positiv auf den Zahnarztbesuch einzustimmen hat sich als hilfreich erwiesen. Besonders wichtig sind diese Interventionen bei Eltern, die selber den Zahnarztbesuch als sehr unangenehm und angstbesetzt erleben [Fayle und Tahmassebi, 2003; Law und Blain, 2003]. Wichtig für die zahnmedizinische Behandlung von Kindern ist außerdem eine kindgerechte verbale und nonverbale Interaktion zwischen Zahnarzt und Kind [zur Übersicht siehe Clinical Guidelines on Behavior Guidance for the Pediatric Dental Patient, American Academy of Pediatric Dentistry, 2005; Fayle und Tahmassebi, 2003]. Diese sollte beinhalten: Eine klare Sprache (freundliche klare Aufforderungen und Informationsweitergabe); Kindgerechte interessante und positive Begriffe für verwendete Gegenstände und Methoden (z.B. «den Zahn mit einem Zaubermittel einschlafen laßen“, anstatt «ein lokales Betäubungsmittel zu geben"); Positive Rückmeldung geben für jedes gezeigte Verhalten das erwünscht ist oder in die erwünschte Richtung geht (z.B. loben, dass das Kind sich auf den Stuhl gesetzt hat, auch wenn es den Mund noch nicht öffnet). Die positive Rückmeldung (Verstärkung) kann in Richtung des Kindes verbal oder auch nonverbal (z.B. Schulterklopfen) gegeben werden, aber auch über das Kind in Richtung Eltern und Praxismitarbeitende geäußert werden; Verwenden der Technik «erklären, zeigen, machen“ («tell, show, do»), bei der alle zahnärztlichen Techniken zuerst dem Kind in verständlicher Sprache erklärt werden, dann in Annäherung, z.B. an einem Modell gezeigt werden und erst dann durchgeführt werden [siehe auch Fayle und Tahmassebi, 2003; Law und Blain, 2003]. Weitere Techniken, die sich positiv auf den Behandlungsverlauf auswirken haben zum Ziel die Kontrolle des Kindes zu erhöhen. Zum Beispiel kann mit dem 
Kind ein Stop-Signal vereinbart werden (Hand heben). Dieses Signal sollte vor Beginn der Behandlung mit dem Kind kurz eingeübt werden [Fayle und Tahmassebi, 2003]. Ablenkung (z.B. Lippenziehen, Musik, Videos, usw.,) hilft die Aufmerksam des Kindes von unangenehmen Aspekten der Behandlung abzuziehen, wobei bisher keine vertiefte Evaluation der Wirksamkeit dieser Methoden vorliegt [Fayle und Tahmassebi, 2003]; Bei ängstlichen Kindern kann Modellernen indiziert sein, indem sie Geschwistern oder Freunden (eventuell auch Videofilmen) zusehen, die kein ängstliches Verhalten während des Zahnarztbesuches zeigen [Fayle und Tahmassebi, 2003]. Auch systematische Desensibilisierung ist bei jüngeren Kindern effektiv [siehe Fayle und Crawford 1997].

Falls ein Kind nicht das gewünschte und für die zahnmedizinische Intervention notwendige Verhalten zeigt, sollte zuerst über positive Verstärkung (siehe oben) versucht werden dieses zu etablieren, wobei unerwünschtes aber tolerierbares Verhalten ignoriert wird. Auch ablenkendes Verhalten des Kindes wird ignoriert. Jedoch ist nicht tolerierbarem negativem Verhalten des Kindes schwieriger zu begegnen. In der Literatur wurden für diese Fälle verschiedene, nach heutigem empfinden wohl meist als nur unter ganz bestimmten Bedingungen akzeptabel empfundene disziplinarische Maßnahmen beschrieben, wie «Kontrolle der Mimik, Gestik und Modulation der Sprache“ («Voice control “; z. B. lauter gesprochene Anweisung an das Kind) [Clinical Guidelines on Behavior Guidance for the Pediatric Dental Patient, American Academy of Pediatric Dentistry, 2005; Pinkham et al. 1985] oder «Hand über Mund“ (dem Kind wird die Hand auf den Mund gelegt, bis es das gewünschte Verhalten zeigt) [Clinical Guidelines on Behavior Guidance for the Pediatric Dental Patient, American Academy of Pediatric Dentistry, 2005; Acs et al., 2001]. Ziel dieser Maßnahmen sei primär, die Aufmerksamkeit des Kindes zu erlangen, um es zu adäquaten Verhalten anhalten zu können [Law und Blain, 2003]. Es wird darauf hingewiesen, dass sichergestellt sein muss, dass das Kind potentiell in der Lage ist das erwünschte Verhalten zu zeigen, durch die Technik keinen Schaden erlangt, dass die Erziehungsberechtigten über die Technik aufgeklärt wurden und ihre Einwilligung dazu gegeben haben. «Hand über Mund“ solle nicht bei primär ängstlichen Kindern eingesetzt werden. Auch wenn diese Technik noch unter bestimmten Bedingungen empfohlen wird [Clinical Guidelines on Behavior Guidance for the Pediatric Dental Patient, American Academy of Pediatric Dentistry, 2005], zeigt sich jedoch, dass sie gesellschaftlich und aus ethischen Gründen nicht mehr oder nur noch in ganz besonderen Ausnahmefällen tolerierbar ist [Havelka et al. 1992; Lawrence et al. 1991; Murphy et al. 1984]. Auch besteht eine bedeutsame Gefahr des Missbrauchs solcher Techniken [Acs et al. 2001; Rayman, 1990]. Ähnliches gilt für disziplinarische Maßnahmen, die das Kind in seiner Bewegung einschränken (festhalten oder fixieren des Kindes) [Law und Blain, 2003]. Auf pharmakologische Interventionen, die zum Ziel haben die Behandlung von Kindern mit oppositionelles Verhalten zu ermöglichen, wird in diesem Artikel nicht eingegangen [siehe dazu z.B. Clinical Guidelines on Behavior Guidance for the Pediatric Dental Patient, American Academy of Pediatric Dentistry, 2005; Paterson und Thamassabi, 2003].

\section{Patienten mit Einschränkungen und ältere Menschen}

Bei Patienten mit körperlicher Behinderung muss sichergestellt werden, dass alle zur Mundhygiene und zahnmedizinische Versorgung notwendigen Aktivitäten durchgeführt werden können. Besondere Aufmerksamkeit verlangen Patienten mit geistiger Behinderung. Hier muss individuell, gegebenenfalls in enger Abstimmung mit den Betreuungspersonen, auf mögliche Hindernisse in der Mundhygiene und zahnmedizinischen Versorgung eingegangen werden.

Für alte Menschen erlangen, oft bereits durch die altersbedingte Verschlechterung des Zahnstatus und die dadurch wahrgenommenen Einschränkungen, zahnmedizinische Fragen zunehmend an Bedeutung. Hinzu kommen mit Zahnprothesen einhergehende Umstellungen im Alltagsleben. Es konnte gezeigt werden, dass Selbsthilfegruppen für manche Patienten dabei entlastend sein können [Fiske et al., 1995]. Auch funktionelle Einschränkungen, wie verschlechterte Sehfähigkeit, können zu nachlassender Mundhygiene und nicht rechtzeitiger Erkennung von zahnmedizinischen Erkrankungen führen [Schembri und Fiske, 2001]. Weiter können im Alter auftretende negative Lebensereignisse das Risiko für Parodontalerkrankungen erhöhen [Hugoson et al., 2002]. Neben einer individuellen Adaptation von Behandlung und Prophylaxemaßnahmen an den ältern Menschen können biobehaviorale präventive Gesundheitsprogramme durchgeführt werden, deren positive Effekte für die Mundgesundheit nachgewiesen sind [für eine detaillierte Beschreibung, siehe Persson et al., 1998].

\section{Verhaltensmedizinische Aspekte der zahnmedizinischen Anamnese und Behandlung}

\section{Zahnbehandlungsangst, Zahnbehandlungsphobie}

Mit Zahnbehandlungsangst werden Ängste vor der zahnmedizinischen Behandlung oder damit verbundenen Stimuli beschrieben, von der mindestens jeder sechste Patient berichtet. Unter Zahnarztphobie oder Zahnbehandlungsphobie (selten auch Oralphobie genannt) versteht man stark ausgeprägte Ängste (Prävalenz: zirka $10-20 \%$ ), die mit Vermeidung des Zahnarztbesuches (Prävalenz: 2\%) oder ertragen der Behandlung nur unter höchster Belastung einhergehen können [Colgate, 2004; Francis und Stanley, 1990; Hallstrom und Halling, 1984; Locker et al., 1999; Locker et al., 2001; Thomson et al., 2000]. Zahnbehandlungsphobie wird nach ICD-10 unter F40,2 als spezifische Phobie kodiert [Dilling und World Health Organization, 2000]. Die Prävalenz für Zahnbehandlungsangst ist in den letzten 50 Jahren in den U.S.A. konstant geblieben, während allgemeine Ängstlichkeit deutlich zugenommen hat [Smith und Heaton, 2003]. Als Ursachen für Zahnbehandlungsphobie werden immer wieder traumatische Erlebnisse während Behandlungen genannt, was auf die 
Möglichkeit eines prophylaktischen Beitrags zur Entstehung von Zahnbehandlungsphobie durch Vermeidung traumatischer Wahrnehmungen von Zahnbehandlungen, z.B. durch adäquate Informationsvermittlung vor der Behandlung von zahnmedizinischer Seite, hinweist [Lindsay und Jackson, 1993]. Andere Studien finden jedoch keinen Zusammenhang zwischen Zahnbehandlungsphobien im jungen Erwachsenenalter und negativen Zahnbehandlungsverfahren [Thomson et al., 2000]. Aversive Lebenserfahrungen, wie sexuelle Gewalt während der Kindheit oder im Erwachsenenalter gehen mit erhöhter Zahnbehandlungsangst einher [Walker et al., 1996]. So zeigen fünfundsiebzig Prozent aller Frauen mit sexuellen Gewalterfahrungen, die mit oraler Penetration einhergingen, starke Zahnbehandlungsangst [Willumsen, 2001, 2004].

Zur Diagnostik von Zahnbehandlungsphobie liegen spezifische Fragebögen vor (zum Beispiel dental anxiety scale (DAS) [Corah, 1969], deutsche Version: [Margraf-Stiksrud, 1996]). Therapeutisch eingesetzt werden sowohl angstlösende und schmerzlindernde Medikamente, als auch Psychotherapie. In seltenen Fällen kann es notwendig sein, die Zahnbehandlung unter anxiolytischen oder sedierenden Medikamenten oder sogar Vollnarkose durchzuführen. Zur Behandlung der Zahnbehandlungsphobie stellen psychotherapeutische Methoden die Verfahren der ersten Wahl dar. Zum Einsatz kommen Techniken des Beobachtungslernens, Konfrontationsverfahren in vivo und in sensu, Entspannungsverfahren, kognitive Verfahren und Kombinationen aus den genannten Ansätzen. Eine aktuelle Metaanalyse zeigt, dass behaviorale Interventionen Zahnbehandlungsangst nachhaltig deutlich reduzieren (mittlere bis große Effektstärken) und bei der Mehrzahl der Teilnehmenden auch langfristig zu regelmäßigen Zahnarztbesuchen führt [Kvale et al., 2004]. Verschiedene Ansätze zur Anwendung von Hypnose in der Zahnmedizin, z.B. bei Schmerzen oder Behandlungsangst liegen vor, auf die hier jedoch nicht näher eingegangen werden soll [siehe Deutsche Gesellschaft für Zahnärztliche Hypnose e.V., 2005; Patel et al., 2000; Shaw und Niven, 1996].

\section{Schlussbemerkungen und Ausblick}

Auf weitere verhaltensmedizinische und psychologische Aspekte, die für die Zahnheilkunde von Relevanz sind (z.B. bei Mundtrockenheit, Geschmacksstörungen, Amalgamintoleranz, Krebserkrankungen im Mundbereich (z.B. Mundhölenkarzinome), persistierenden idiopathischen Zahnschmerzen, Bruxismus, Schmerzen bei der zahnärztlichen Behandlung, Zahnarzt-Patient-Kommunikation, Belastungen im Zusammenhang mit zahnmedizinischen Berufen sowie bei Problemstellungen spezieller Disziplinen, wie Kieferorthopädie, Mund-Kiefer-Gesichts-Chirurgie, Prothetik (zum Beispiel Daumenlutschen, psychogene Zahnprothesenintoleranz oder psychosoziale Probleme bei Kiefergesichtsversehrten) kann hier leider aus Platzgründen nicht genauer eingegangen werden, so dass auf die vorhandene, teils ausführliche Literatur verwiesen wird [zu Bruxismus siehe z.B. Korn 2005].

Einige der in diesem Artikel dargestellten zahnmedizinischen Beschwerdebilder sind dadurch gekennzeichnet, dass kein ausreichender organischer Befund vorliegt. Außerdem verstärken sie sich durch Belastungen und können nicht durch ansonsten wirksame klassisch medizinische Maßnahmen gelindert werden. Deswegen werden sie auch unter dem Begriff «psychosomatisch bedingte Beschwerden im Mund-Kiefer und Gesichtsbereich“ zusammenfasst, der Parallelen mit der Diagnosegruppe «Somatoforme Störungen“ aufweist [Dilling und World Health Organization, 2000].

Aus unseren Ausführungen wird deutlich, dass das umfangreiche Wissen an der Schnittstelle von Verhaltensmedizin, Psychotherapie und Zahnheilkunde vielfältige Ansatzmöglichkeiten für klinische Interventionen bietet. Die Wirksamkeit der sich dabei ergebenden Verfahren ist zum Teil ausreichend belegt. In einigen Bereichen stehen jedoch Effektivitäts- und Effizienzstudien, welche wissenschaftlichen Gütekriterien genügen, noch aus. Ein zunehmendes Verständnis psychobiologischer Prozesse (zum Beispiel spezifische Dysregulationen in Hormon- oder Neurotransmittersystemen), die an der Schnittstelle zwischen Psyche, Verhalten und Soma bei der Entstehung und Aufrechterhaltung zahnmedizinischer Beschwerden relevant sind, ermöglicht neue Perspektiven für Prävention und Intervention dieser Erkrankungen (exemplarisch in Abbildung 1 dargestellt). Klaus Grawe [2004] stellte in seinem Buch „Neuropsychotherapie“ umfassend dar, wie sich Psychotherapie basierend auf biologischen Überlegungen konzeptualisieren lässt. Zusammen mit einem wachsenden Wissen um psychobiologische Prozesse bei zahnmedizinischen Erkrankungen, könnte dies womöglich eine Grundlage für die Entwicklung spezifischer „neuropsychotherapeutischer“ Maßnahmen bei zahnmedizinischen Erkrankungen bieten. In den letzten Jahren haben wir bereits eine Menge an Wissen in diesem Bereich hinzu gewonnen. Von der Zukunft bleibt zu hoffen, dass dieses Wissen weiter anwächst und dass vermehrte Anstrengungen getätigt werden, um die gewonnen Erkenntnisse zum Nutzen der aktuell und zukünftig Betroffenen in die klinische Praxis umzusetzen.

\section{Dank}

Die Autoren möchten sich herzlich bedanken, bei Frau Dr. rer. nat. Elisabeth Gläßer, Herrn Dr. med. dent. Michael Erbshäuser und Frau Dorothée Bentz für die Durchsicht einer früheren Version des Manuskripts und hilfreiche Kommentare und bei Torsten Hero für die Mithilfe bei der Manuskripterstellung. Der Erstautor wurde gefördert durch ein Stipendium des Cusanuswerks. 


\section{Literatur}

Acs G, Hersch G, Testen RD, Ng MW: A 20-year perspective on the changing use of hand over mouth (HOM) and restraint in postdoctoral pediatric dental education. Pediatr Dent 2001;23:301-306.

American Academy of Pediatric Dentistry: Clinical Guidelines on behavior guidance for the pediatric dental patient. Verfügbar unter: http://www.aapd.org/media/Policies_Guidelines/G_BehavGuide.pdf [02/11 2005], (2005).

Al-Zahrani MS, Bissada NF, Borawskit EA: Obesity and periodontal disease in young, middle-aged, and older adults. J Periodontol 2003;74(5):610-615.

Baker PJ, Roopenian DC: Genetic susceptibility to chronic periodontal disease. Microbes

Infect 2002;4(11):1157-1167.

Ben-Aryeh H, Horowitz G, Nir D, Laufer D: Halitosis: an interdisciplinary approach. Am J Otolaryngol 1998;19(1):8-11.

Bergdahl M, Bergdahl J: Burning mouth syndrome: prevalence and associated factors. J Oral Pathol Med 1999;28(8):350-354.

Bogetto F, Maina G, Ferro G, Carbone M, Gandolfo S: Psychiatric comorbidity in patients with burning mouth syndrome. Psychosom Med 1998;60(3):378-385.

Bosch JA, de Geus EJ, Veerman EC, Hoogstraten J, Nieuw Amerongen AV: Innate secretory immunity in response to laboratory stressors that evoke distinct patterns of cardiac autonomic activity. Psychosom Med 2003;65(2):245-258.

Bosch JA, Turkenburg M, Nazmi K, Veerman EC, de Geus EJ, Nieuw Amerongen AV: Stress as a determinant of saliva-mediated adherence and coadherence of oral and nonoral microorganisms. Psychosom Med 2003;65(4):604-612.

Bosy A: Oral malodor: philosophical and practical aspects. J Can Dent Assoc 1997;63(3):196-201.

Breivik T, Opstad PK, Gjermo P, Thrane PS: Effects of hypothalamic-pituitary-adrenal axis reactivity on periodontal tissue destruction in rats. Eur J Oral Sci 2000;108(2):115-122.

Breivik T, Rook GA: Prevaccination with SRL172 (heat-killed Mycobacterium vaccae) inhibits experimental periodontal disease in Wistar rats. Clin Exp Immunol 2000;120(3):463-467.

Breivik T, Sluyter F, Hof M, Cools A: Differential susceptibility to periodontitis in genetically selected Wistar rat lines that differ in their behavioral and endocrinological response to stressors. Behav Genet 2000;30(2):123-130.

Breivik T, Stephan M, Brabant GE, Straub RH, Pabst R, von Horsten S: Postnatal lipopolysaccharide-induced illness predisposes to periodontal disease in adulthood. Brain Behav Immun 2002;16(4):421-438.

Breivik T, Thrane PS: Psychoneuroimmune interactions in periodontal disease; in Ader R, Felten DL, Cohen N (eds): Psychoneuroimmunology (3 ed). San Diego, Academic Press, 2001, vol 2, pp 627-644.

Breivik T, Thrane PS, Gjermo P, Cools A, Myhrer T: Effects of hippocampal lesioning on experimental periodontitis in Wistar rats. J Periodontal Res 2002;37(5):360-365.

Breivik T, Thrane PS, Gjermo P, Fonnum F: Postnatal glutamate-induced central nervous system lesions alter periodontal disease susceptibility in adult Wistar rats. J Clin Periodontol 2001;28(10):904-909.

Breivik T, Thrane PS, Gjermo P, Opstad PK: Glucocorticoid receptor antagonist RU 486 treatment reduces periodontitis in Fischer 344 rats. J Periodontal Res 2000;35(5):285-290.

Broadbent JM, Ayers KM, Thomson WM: Is attention-deficit hyperactivity disorder a risk factor for dental caries? A case-control study. Caries Res 2004;38(1):29-33.

Christensen GJ: Oral care for patients with bulimia. J Am Dent Assoc 2002;133(12):1689-1691.

Cohen LK: Dentistry and the behavioral/social sciences: an historical overview. J Behav Med 1981;4(3):247-256.

Coil JM, Yaegaki K, Matsuo T, Miyazaki H: Treatment needs (TN) and practical remedies for halitosis. Int Dent J 2002;52 Suppl $3187-191$.

Colgate: Emnid-Umfrage. 2004.

Corah NL: Development of a dental anxiety scale. J Dent Res 1969;48(4):596.

Croucher R, Marcenes WS, Torres MC, Hughes F, Sheiham A: The relationship between life-events and periodontitis. A case-control study. J Clin Periodontol 1997;24(1):39-43.

Cutress TW: Periodontal health and periodontal disease in young people: global epidemiology. Int Dent J 1986;36(3):146-152.

Deinzer R, Forster P, Fuck L, Herforth A, Stiller-Winkler R, Idel H: Increase of crevicular interleukin 1beta under academic stress at experimental gingivitis sites and at sites of perfect oral hygiene. J Clin Periodontol 1999;26(1):1-8.

Deinzer R, Granrath N, Stuhl H, Twork L, Idel H, Waschul B, Herforth A: Acute stress effects on local Il-1beta responses to pathogens in a human in vivo model. Brain Behav Immun 2004;18(5):458-467.

Deinzer R, Hilpert D, Bach K, Schawacht M, Herforth A: Effects of academic stress on oral hygiene--a potential link between stress and plaque-associated disease? J Clin Periodontol 2001;28(5):459-464.

Deinzer R, Kleineidam C, Stiller-Winkler R, Idel H, Bachg D: Prolonged reduction of salivary immunoglobulin A (sIgA) after a major academic exam. Int J Psychophysiol 2000a;37(3):219-232.

Deinzer R, Kottmann W, Forster P, Herforth A, Stiller-Winkler R, Idel H: After-effects of stress on crevicular interleukin-1beta. J Clin Periodontol 2000;27(1):74-77.

Deinzer R, Ruttermann S, Mobes O, Herforth A: Increase in gingival inflammation under academic stress. J Clin Periodontol 1998;25(5):431-433.

Deinzer R, Schuller N: Dynamics of stress-related decrease of salivary immunoglobulin A (sIgA): relationship to symptoms of the common cold and studying behavior. Behav Med 1998a;23(4):161-169.

Deutsche Gesellschaft für Zahnärztliche Hypnose e.V.: Homepage. Verfügbar unter: http://www.dgzh.de/ [08/02 2005], (2005).

Dilling H, World Health Organization: Internationale Klassifikation psychischer Störungen ICD-10 Kapitel V (F) klinisch-diagnostische Leitlinien (4., durchges. u. erg. Aufl.), Huber, 2000.

Dworkin SF: Behavioral dentistry: a renewed paradigm for the future. J Indiana Dent Assoc 2000;79(4):11-15.

Eli I, Baht R, Kozlovsky A, Rosenberg M: The complaint of oral malodor: possible psychopathological aspects. Psychosom Med 1996;58(2):156-159.

Fayle SA, Crawford PJM: Making dental treatment acceptable to children. Dent Profile 1997; September:18-22.

Fayle SA, Tahmassebi JF: Paediatric dentistry in the new millennium: 2. Behaviour Management - Helping Children to accept dentistry. Pediatr Dent 2003;30(6):294-298.

Fiske J, Davis DM, Horrocks P: A self-help group for complete denture wearers. Br Dent J 1995;178(1):18-22.

Francis RD, Stanley GV: Estimating the prevalence of dental phobias. Aust Dent J 1990;35(5):449-453.

Freire M, Hardy R, Sheiham A: Mothers' sense of coherence and their adolescent children's oral health status and behaviours. Community Dent Health 2002;19(1):24-31.

Friedlander AH, Mahler ME: Major depressive disorder. Psychopathology, medical management and dental implications. J Am Dent Assoc 2001;132(5):629638.

Friedlander AH, Marder SR, Sung EC, Child JS: Panic disorder: psychopathology, medical management and dental implications. J Am Dent Assoc 2004;135(6):771-778; quiz 796-777.

Genco RJ, Ho AW, Grossi SG, Dunford RG, Tedesco LA: Relationship of stress, distress and inadequate coping behaviors to periodontal disease. J Periodontol 1999;70(7):711-723.

Genco RJ, Ho AW, Kopman J, Grossi SG, Dunford RG, Tedesco LA: Models to evaluate the role of stress in periodontal disease. Ann Periodontol 1998;3(1):288-302.

Giannopoulou C, Kamma JJ, Mombelli A: Effect of inflammation, smoking and stress on gingival crevicular fluid cytokine level. J Clin Periodontol 2003;30(2):145-153. 
Grawe K: Neuropsychotherapie. Göttingen, Hogrefe, 2004.

Gurenlian JR: Eating disorders. J Dent Hyg 2002;76(3):219-234; quiz 236-217.

Hagelberg N, Forssell H, Rinne JO, Scheinin H, Taiminen T, Aalto S, Luutonen S, Nagren K, Jaaskelainen S: Striatal dopamine D1 and D2 receptors in burning mouth syndrome. Pain 2003;101(1-2):149-154.

Hakeberg M, Berggren U, Hagglin C, Ahlqwist M: Reported burning mouth symptoms among middle-aged and elderly women. Eur J Oral Sci 1997;105(6):539543.

Hallstrom T, Halling A: Prevalence of dentistry phobia and its relation to missing teeth, alveolar bone loss and dental care habits in an urban community sample. Acta Psychiatr Scand 1984;70(5):438-446.

Havelka C, McTigue D, Wilson S, Odom J: The influence of social status and prior explanation on parental attitudes toward behavior management techniques. Pediatr Dent 1992;14:376-381.

Hildebrand HC, Epstein J, Larjava H: The influence of psychological stress on periodontal disease. J West Soc Periodontol Periodontal Abstr 2000;48(3):69-77.

Hugoson A, Jordan T: Frequency distribution of individuals aged 20-70 years according to severity of periodontal disease. Community Dent Oral Epidemiol 1982;10(4):187-192.

Hugoson A, Ljungquist B, Breivik T: The relationship of some negative events and psychological factors to periodontal disease in an adult Swedish population 50 to 80 years of age. J Clin Periodontol 2002;29(3):247-253.

Jaaskelainen SK, Rinne JO, Forssell H, Tenovuo O, Kaasinen V, Sonninen P, Bergman J: Role of the dopaminergic system in chronic pain -- a fluorodopa-PET study. Pain 2001;90(3):257-260.

Kamma JJ, Giannopoulou C, Vasdekis VG, Mombelli A: Cytokine profile in gingival crevicular fluid of aggressive periodontitis: influence of smoking and stress. J Clin Periodontol 2004;31(10):894-902.

Korn HJ: Biofeedback und zahnmedizinische Behandlungsansätze bei temporomandibulären Störungen und Bruxismus. Verhaltenstherapie 2005; 15:94-102.

Kvale G, Berggren U, Milgrom P: Dental fear in adults: a meta-analysis of behavioral interventions. Community Dent Oral Epidemiol 2004;32(4):250-264.

Lamey PJ, Freeman R, Eddie SA, Pankhurst C, Rees T: Vulnerability and presenting symptoms in burning mouth syndrome. Oral Surg Oral Med Oral Pathol Oral Radiol Endod 2005;99(1):48-54.

Law CS, Blain S: Approaching the pediatric dental patient: A Review of nonpharmacological behavior management strategies. J Calif Dent Assoc 2003;31(9):703-713.

Lawrence SM, McTigue DJ, Wilson S, Odom JG, Waggoner WF, Fields HW Jr. : Parental attitudes toward behavior management techniques used in pediatric dentistry. Padiatr Dent 1991;13:151-155.

Lenton P, Majerus G, Bakdash B: Counseling and treating bad breath patients: a step-by-step approach. J Contemp Dent Pract 2001;2(2):46-61.

LeResche L, Dworkin SF: The role of stress in inflammatory disease, including periodontal disease: review of concepts and current findings. Periodontol 2000 2002;3091-103.

Linden GJ, Mullally BH, Freeman R: Stress and the progression of periodontal disease. J Clin Periodontol 1996;23(7):675-680.

Lindsay SJ, Jackson C: Fear of routine dental treatment in adults: Its nature and management. Psychology and Health 1993;8(2-3):135-153.

List T, Axelsson S, Leijon G: Pharmacologic interventions in the treatment of temporomandibular disorders, atypical facial pain, and burning mouth syndrome. A qualitative systematic review. J Orofac Pain 2003;17(4):301-310.

Locker D, Liddell A, Shapiro D: Diagnostic categories of dental anxiety: a population-based study. Behav Res Ther 1999;37(1):25-37.

Locker D, Poulton R, Thomson WM: Psychological disorders and dental anxiety in a young adult population. Community Dent Oral Epidemiol 2001;29(6):456463.

Longley AJ, Doyle PE: Mental illness and the dental patient. J Dent Hyg 2003;77(3):190-204; quiz 204-196.

Malki GA, Zawawi KH, Melis M, Hughes CV: Prevalence of bruxism in children receiving treatment for attention deficit hyperactivity disorder: a pilot study. J Clin Pediatr Dent 2004;29(1):63-67.

Marcenes WS, Sheiham A: The relationship between work stress and oral health status. Soc Sci Med 1992;35(12):1511-1520.

Margraf-Stiksrud J: Angst und Angstabbau; in Sergl HG (ed), Psychologie und Psychosomatik in der Zahnheilkunde, Urban und Schwarzenberg, 1996.

Meisel P, Schwahn C, Gesch D, Bernhardt O, John U, Kocher T: Dose-effect relation of smoking and the interleukin-1 gene polymorphism in periodontal disease. J Periodontol 2004;75(2):236-242.

Meisel P, Siegemund A, Dombrowa S, Sawaf H, Fanghaenel J, Kocher T: Smoking and polymorphisms of the interleukin-1 gene cluster (IL-1alpha, IL-1beta, and IL-1RN) in patients with periodontal disease. J Periodontol 2002;73(1):27-32.

Meisel P, Siegemund A, Grimm R, Herrmann FH, John U, Schwahn C, Kocher T: The interleukin-1 polymorphism, smoking, and the risk of periodontal disease in the population-based SHIP study. J Dent Res 2003;82(3):189-193.

Mengel R, Bacher M, Flores-De-Jacoby L: Interactions between stress, interleukin-1beta, interleukin- 6 and cortisol in periodontally diseased patients. J Clin Periodontol 2002;29(11):1012-1022.

Merchant AT, Pitiphat W, Ahmed B, Kawachi I, Joshipura K: A prospective study of social support, anger expression and risk of periodontitis in men. J Am Dent Assoc 2003;134(12):1591-1596.

Monteiro da Silva AM, Oakley DA, Newman HN, Nohl FS, Lloyd HM: Psychosocial factors and adult onset rapidly progressive periodontitis. J Clin Periodontol 1996;23(8):789-794.

Murata T, Yamaga T, Iida T, Miyazaki H, Yaegaki K: Classification and examination of halitosis. Int Dent J 2002;52 Suppl 3181-186.

Murphy MG, Fields HW Jr., Machen JB: Parental acceptance of pediatric dentistry behavior management techniques. Pediatr Dent 1984;6:193-198.

Patel B, Potter C, Mellor AC: The use of hypnosis in dentistry: a review. Dent Update 2000;27(4):198-202.

Paterson SA, Thamassebi JF: Paediatric dentistry in the new millennium: 3. Use of inhalation sedation in paediatric dentistry. Dent Update 2003;30(7):350-356.

Persson RE, Persson GR, Powell LV, Kiyak HA: Periodontal effects of a biobehavioral prevention program. J Clin Periodontol 1998;25(4):322-329.

Petersen PE: The World Oral Health Report 2003: Continuous improvement of oral health in the 21st century - the approach of the WHO Global Oral Health Programme. Geneva, Switzerland, Oral Health Programme, Noncommunicable Disease Prevention and Health Promotion, World Health Organization, 2003.

Pine CM, McGoldrick PM, Burnside G, Curnow MM, Chesters RK, Nicholson J, Huntington E: An intervention programme to establish regular toothbrushing: understanding parents' beliefs and motivating children. Int Dent J 2000;Suppl Creating A Successful :312-323.

Pinkham JR, Paterson JR: Voice control: an old technique reexamined. ASCD J Dent Child 1985;52:199-202.

Quinonez RB, Keels MA, Vann WF, Jr., McIver FT, Heller K, Whitt JK: Early childhood caries: analysis of psychosocial and biological factors in a high-risk population. Caries Res 2001;35(5):376-383.

Quirynen M, Zhao H, van Steenberghe D: Review of the treatment strategies for oral malodour. Clin Oral Investig 2002;6(1):1-10.

Rayman MS: Hand-over-mouth technique: acceptable in the 1990s? J Calif Dent Assoc 1990;18:65-67.

Reich E: Trends in caries and periodontal health epidemiology in Europe. Int Dent J 2001;51(6 Suppl 1):392-398.

Reisine ST, Psoter W: Socioeconomic status and selected behavioral determinants as risk factors for dental caries. J Dent Educ 2001;65(10):1009-1016.

Restrepo CC, Alvarez E, Jaramillo C, Velez C, Valencia I: Effects of psychological techniques on bruxism in children with primary teeth. J Oral Rehabil 2001;28(4):354-360

Richter JL: Diagnosis and treatment of halitosis. Compend Contin Educ Dent 1996;17(4):370-372, 374-376 passim; quiz 388.

Roldan S, Herrera D, Sanz M: Biofilms and the tongue: therapeutical approaches for the control of halitosis. Clin Oral Investig 2003;7(4):189-197.

Sanz M, Roldan S, Herrera D: Fundamentals of breath malodour. J Contemp Dent Pract 2001;2(4):1-17.

Scala A, Checchi L, Montevecchi M, Marini I, Giamberardino MA: Update on burning mouth syndrome: overview and patient management. Crit Rev Oral Biol Med 2003;14(4):275-291.

Schembri A, Fiske J: The implications of visual impairment in an elderly population in recognizing oral disease and maintaining oral health. Spec Care Dentist 2001;21(6):222-226 
Shapira L, Frolov I, Halabi A, Ben-Nathan D: Experimental stress suppresses recruitment of macrophages but enhanced their P. gingivalis LPS-stimulated secretion of nitric oxide. J Periodontol 2000;71(3):476-481.

Shaw AJ, Niven N: Theoretical concepts and practical applications of hypnosis in the treatment of children and adolescents with dental fear and anxiety. Br Dent J 1996;180(1):11-16

Sluyter F, Breivik T, Cools A: Manipulations in maternal environment reverse periodontitis in genetically predisposed rats. Clin Diagn Lab Immunol 2002;9(4):931-932.

Smith TA, Heaton LJ: Fear of dental care: are we making any progress? J Am Dent Assoc 2003;134(8):1101-1108.

Sutton PR: The early onset of acute dental caries in adults following mental stress. N Y State Dent J 1965;31(10):450-456.

Tangerman A: Halitosis in medicine: a review. Int Dent J 2002;52 Suppl 3201-206.

Thomson WM, Locker D, Poulton R: Incidence of dental anxiety in young adults in relation to dental treatment experience. Community Dent Oral Epidemiol 2000;28(4):289-294.

Tickle M, Brown P, Blinkhorn A, Jenner T: Comparing the ability of different area measures of socioeconomic status to segment a population according to caries prevalence. Community Dent Health 2000;17(3):138-144.

Tickle M, Williams M, Jenner T, Blinkhorn A: The effects of socioeconomic status and dental attendance on dental caries' experience, and treatment patterns in 5-year-old children. Br Dent J 1999;186(3):135-137.

Tsai C, Hayes C, Taylor GW: Glycemic control of type 2 diabetes and severe periodontal disease in the US adult population. Community Dent Oral Epidemiol 2002;30(3):182-192.

Walker EA, Milgrom PM, Weinstein P, Getz T, Richardson R: Assessing abuse and neglect and dental fear in women. J Am Dent Assoc 1996;127(4):485-490.

Waschul B, Herforth A, Stiller-Winkler R, Idel H, Granrath N, Deinzer R: Effects of plaque, psychological stress and gender on crevicular Il-1beta and Il-1ra secretion. J Clin Periodontol 2003;30(3):238-248.

Weinstein P, Harrison R, Benton T: Motivating parents to prevent caries in their young children: one-year findings. J Am Dent Assoc 2004;135(6):731-738.

Willumsen T: Dental fear in sexually abused women. Eur J Oral Sci 2001;109(5):291-296.

Willumsen T: The impact of childhood sexual abuse on dental fear. Community Dent Oral Epidemiol 2004;32(1):73-79.

Yaegaki K, Coil JM: Genuine halitosis, pseudo-halitosis, and halitophobia: classification, diagnosis, and treatment. Compend Contin Educ Dent 2000;21(10A):880-886, 888-889; quiz 890.

Zakrzewska JM, Forssell H, Glenny AM: Interventions for the treatment of burning mouth syndrome: a systematic review. J Orofac Pain 2003;17(4):293-300.

Zakrzewska JM, Glenny AM, Forssell H: Interventions for the treatment of burning mouth syndrome. Cochrane Database Syst Rev 2001;(3):CD002779. 
Legenden

zu Meinlschmidt \& Bolt: „Verhaltensmedizin, Psychotherapie und Zahnheilkunde“

Tabelle 1: Zahnmedizinische Erkrankungen, deren wichtigste Ursachen und Risikofaktoren, relevante psychobiologische Befunde und Ansätze für verhaltensmedizinische Interventionen

Tabelle 2: Die am Häufigsten beschriebenen psychischen Störungen mit Bezug zur Zahnmedizin

Abbildung 1: Biopsychosoziales Modell der Entstehung und Aufrechterhaltung von Parodontitis 
Tabelle 2: Die am Häufigsten beschriebenen psychischen Störungen mit Bezug zur Zahnmedizin

\begin{tabular}{|c|c|}
\hline Psychische Störung & Bezug zu Zahnmedizin \\
\hline Major Depression & $\begin{array}{l}\text { - Somatisierungsneigung, einseitige Ernährung, mangelnde Hygiene und } \\
\text { Antidepressiva können zu zahnmedizinischen Problemen führen } \\
\text { - Depressive suchen womöglich zahnmedizinische Behandlung auf, bevor sie } \\
\text { auf psychische Problematik aufmerksam werden } \\
\text { - Wo immer möglich, sollte zahnmedizinische Behandlung nach } \\
\text { Einverständnis des Patienten in Rücksprache mit dem behandelnden } \\
\text { Psychiater oder Psychotherapeuten erfolgen }\end{array}$ \\
\hline Angststörungen & $\begin{array}{l}\text { - Anxiolytische Medikamente können zahnmedizinische Probleme } \\
\text { begünstigen }\end{array}$ \\
\hline $\begin{array}{l}\text { Erschöpfungssyndrom } \\
\text { (Chronic fatigue syndrom) }\end{array}$ & 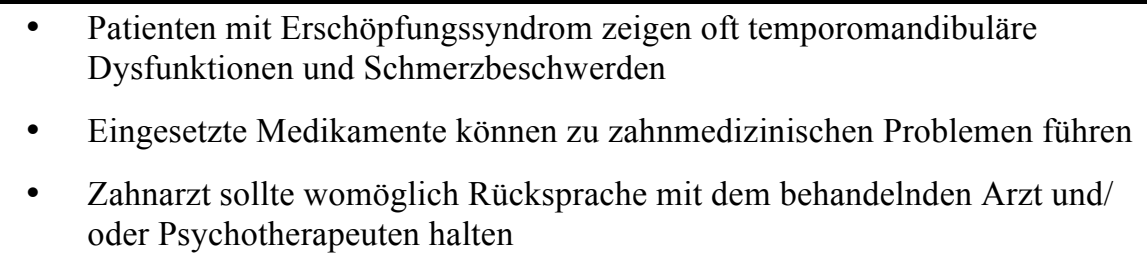 \\
\hline $\begin{array}{l}\text { Essstörungen } \\
\text { (Anorexia nervosa, Bulimia } \\
\text { nervosa) }\end{array}$ & $\begin{array}{l}\text { - Gehäuft zahnmedizinische Probleme durch Mangelzustände und durch die } \\
\text { durch Erbrechen auftretenden Schäden durch Magensäure im Mundbereich } \\
\text { - } \quad \text { Interdisziplinäre Behandlung ist notwendig }\end{array}$ \\
\hline $\begin{array}{l}\text { Aufmerksamkeitshyper- } \\
\text { aktivitätsstörung bei Kindern }\end{array}$ & $\begin{array}{l}\text { - } \quad \text { Erhöhtes Risiko für Zahnschäden liegt vor } \\
\text { - Psychostimulantien können womöglich das Auftreten von Bruxismus } \\
\text { fördern }\end{array}$ \\
\hline $\begin{array}{l}\text { Zwanghafte } \\
\text { Persönlichkeitsstörung, } \\
\text { Zwangshandlungen, Manie }\end{array}$ & - $\quad$ Zahnschäden durch exzessives Zähneputzen \\
\hline $\begin{array}{l}\text { Substanzmissbrauch, } \\
\text { Psychotische Störungen }\end{array}$ & $\begin{array}{l}\text { - Zahnschäden als Folge der eingesetzten Medikamente (verminderter } \\
\text { Speichelfluss; Dyskinesien können Mundhygiene beeinträchtigen) } \\
\text { - Halluzinatorische Körperempfindungen (Zönästhesien) im Mundbereich im } \\
\text { Rahmen einer psychotischen Symptomatik können vorliegen }\end{array}$ \\
\hline
\end{tabular}


Tabelle 1: Zahnmedizinische Erkrankungen, deren wichtigste Ursachen und Risikofaktoren, relevante psychobiologische Befunde und Ansätze für verhaltensmedizinische Interventionen

\begin{tabular}{|c|c|c|c|}
\hline $\begin{array}{l}\text { Zahnmedizinische } \\
\text { Erkrankungen } \\
\text { und Symptome }\end{array}$ & $\begin{array}{l}\text { Wichtige Ursachen und } \\
\text { Risikofaktoren }\end{array}$ & Psychobiologie & $\begin{array}{l}\text { Verhaltensmedizinische } \\
\text { Interventionen }^{1}\end{array}$ \\
\hline Karies & $\begin{array}{l}\text { - Mangelhafte Mundhygiene } \\
\text { - Dem Zahnschmelz } \\
\text { anhaftende Bakterien } \\
\text { - Plaques } \\
\text { - Ungesunde Ernährung } \\
\text { - Struktur des Zahnschmelzes, } \\
\text { Zahnform und } \\
\text { Speichelzusammensetzung }\end{array}$ & $\begin{array}{l}\text { - Stress verändert die } \\
\text { Zusammensetzung der } \\
\text { Speichelproteine }\end{array}$ & $\begin{array}{l}\text { - Sicherstellung adäquater } \\
\text { Zahnprophylaxe (gründliche } \\
\text { Zahnreinigung, zahngesunde } \\
\text { Ernährung, Zufuhr von } \\
\text { Fluoriden) } \\
\text { - Sicherstellung der } \\
\text { Inanspruchnahme } \\
\text { ausreichender } \\
\text { zahnmedizinischer } \\
\text { Versorgung } \\
\text { Karies bei Kindern: } \\
\text { - Interventionen gerichtet an } \\
\text { Eltern/ Betreuungspersonen }\end{array}$ \\
\hline Gingivitis & $\begin{array}{l}\text { - Mechanische Einflüsse } \\
\text { - Toxische Einflüsse } \\
\text { - Hormonelle Faktoren } \\
\text { - Nebenwirkung mancher } \\
\text { Medikamente } \\
\text { - Mangelzustände (besonders } \\
\text { Vitamin C) } \\
\text { - Allgemeinerkrankungen } \\
\text { - Gingivale Läsionen }\end{array}$ & $\begin{array}{l}\text { - Zusammenhang: Stress und } \\
\text { Gingivitis } \\
\text { - Stressbedingte mangelnde } \\
\text { Mundhygiene => } \\
\text { Plaquebildung => } \\
\text { Gingivitis } \\
\text { - Stress => veränderte } \\
\text { Freisetzung von } \\
\text { sekretorischen Speichel- } \\
\text { proteinen => verändertes } \\
\text { Anhaften von Mikro- } \\
\text { organismen an die } \\
\text { Mundschleimhäute => } \\
\text { Gingivitis }\end{array}$ & $\begin{array}{l}\text { - Stressbewältigungstraining } \\
\text { - Etablierung adäquater } \\
\text { Mundhygiene } \\
\text { - Sicherstellung einer } \\
\text { rechtzeitigen zahnärztlichen } \\
\text { Behandlung }\end{array}$ \\
\hline
\end{tabular}

\begin{tabular}{|c|c|c|c|}
\hline Parodontitis & $\begin{array}{l}\text { - Chronisch bakterielle } \\
\text { Entzündung } \\
\text { - Gestörte Wechselbeziehung } \\
\text { zwischen natürlicher } \\
\text { Keimbesiedlung der } \\
\text { Mundhöhle und dem } \\
\text { Immunsystem } \\
\text { - Supragingivaler Zahnstein } \\
\text { - Schlechte Mundhygiene } \\
\text { - Vorhergehende Gingivitis } \\
\text { - Rauchen } \\
\text { - Systemische Erkrankungen } \\
\text { - Übergewicht } \\
\text { - Genetische Faktoren }\end{array}$ & $\begin{array}{l}\text { Tierstudien: } \\
\text { - Risikofaktoren: } \\
\text { lebensgeschichtlich frühe } \\
\text { Belastungen, } \\
\text { Veränderungen in } \\
\text { Stresshormonsystemen und } \\
\text { diese regulierende } \\
\text { Hirnstrukturen } \\
\text { - Durch gezielte } \\
\text { physiologische oder } \\
\text { behaviorale Interventionen } \\
\text { kann Parodontitisrisiko } \\
\text { reduziert werden } \\
\text { Humanstudien: } \\
\text { - Zusammenhang: Stress und } \\
\text { Parodontitis } \\
\text { - Erhöhte lokale } \\
\text { Konzentration } \\
\text { verschiedener Zytokine in } \\
\text { Verbindung mit Stress und } \\
\text { reduzierter Mundhygiene }\end{array}$ & $\begin{array}{l}\text { - Stressbewältigungstraining } \\
\text { - Etablierung adäquater } \\
\text { Mundhygiene } \\
\text { - Sicherstellung einer } \\
\text { rechtzeitigen Behandlung } \\
\text { - Wenn möglich, Einbezug } \\
\text { genetischer, physiologischer, } \\
\text { psychosozialer und } \\
\text { behavioraler Informationen } \\
\text { zur Einleitung präventiver } \\
\text { und therapeutische } \\
\text { Maßnahmen }\end{array}$ \\
\hline
\end{tabular}




\begin{tabular}{|c|c|c|c|}
\hline $\begin{array}{l}\text { "Burning-mouth"- } \\
\text { Syndrome }\end{array}$ & $\begin{array}{l}\text { - Ätiologie noch weitgehend } \\
\text { unverstanden } \\
\text { - Oft begleitet von Diabetes, } \\
\text { Anämie, Mangel- } \\
\text { erscheinungen und oralen } \\
\text { Dysfunktionen } \\
\text { - Ausschluss organischer } \\
\text { Ursachen, die die } \\
\text { Beschwerden ausreichend } \\
\text { erklären können (u.a. } \\
\text { bakterielle, mykotische oder } \\
\text { virale Infektionen) }\end{array}$ & $\begin{array}{l}\text { - Vermehrtes Vorhandensein } \\
\text { aversiver } \\
\text { Kindheitserfahrungen } \\
\text { - Veränderungen im } \\
\text { zentralen Dopaminsystem }\end{array}$ & - Kognitive Verhaltenstherapie \\
\hline Mundgeruch & $\begin{array}{l}\text { Geruchsursachen: } \\
\text { - Stoffwechselprodukte } \\
\text { bestimmter gramnegativer } \\
\text { Bakterien } \\
\text { - Mandel- und Kieferhöhlen- } \\
\text { entzündungen } \\
\text { - Pfeiffersches Drüsenfieber } \\
\text { - Krankhafte Veränderungen } \\
\text { der unteren Lungenwege } \\
\text { - Stoffwechselprodukte in } \\
\text { Folge von systemischen } \\
\text { Erkrankungen, } \\
\text { Nahrungsmitteln und } \\
\text { Medikamenten } \\
\text { - Magen- und } \\
\text { Speiseröhrenerkrankungen }\end{array}$ & & $\begin{array}{l}\text { - Wichtig: Unterscheidung } \\
\text { zwischen genuiner Halitose, } \\
\text { Pseudohalitose und } \\
\text { Halitophobie } \\
\text { - Bei genuiner Halitose: } \\
\text { Etablierung adäquater } \\
\text { hygienischer Maßnahmen } \\
\text { - Bei Pseudohalitose: } \\
\text { verhaltensmedizinische } \\
\text { Interventionen ggf. durch } \\
\text { Zahnarzt } \\
\text { - Bei Halitophobie: } \\
\text { Anwendung } \\
\text { psychotherapeutischer } \\
\text { Interventionen durch } \\
\text { Spezialisten } \\
\text { Allgemein: } \\
\text { - Einbezug psychologischer } \\
\text { Informationen bei Anamnese } \\
\text { von Halitosepatienten } \\
\text { - Thematisierung von } \\
\text { Beeinträchtigungen durch } \\
\text { Mundgeruch bereits in } \\
\text { Risikogruppen }\end{array}$ \\
\hline
\end{tabular}

'Die aufgeführten Interventionen leiten sich aus verhaltensmedizinischen Forschungsergebnissen zu zahnmedizinischen Erkrankungen und Beschwerden ab. Es sei darauf hingewiesen, dass einige der genannten Interventionsansätze in ihrer Wirksamkeit (noch) nicht durch kontrollierte Studien abgesichert sind. Neben den verhaltensmedizinischen Interventionen können oder müssen bei einigen Beschwerdebildern natürlich auch zahnmedizinische Interventionen, ggf. auch in deren Kombination zur Anwendung kommen. 
Abbildung 1: Biopsychosoziales Modell der Entstehung und Aufrechterhaltung von Parodontitis

Lifestyle (z.B. Rauchen, Ernährung, Übergewicht), frühe aversive Lebenserfahrungen, psychosozialer Stress

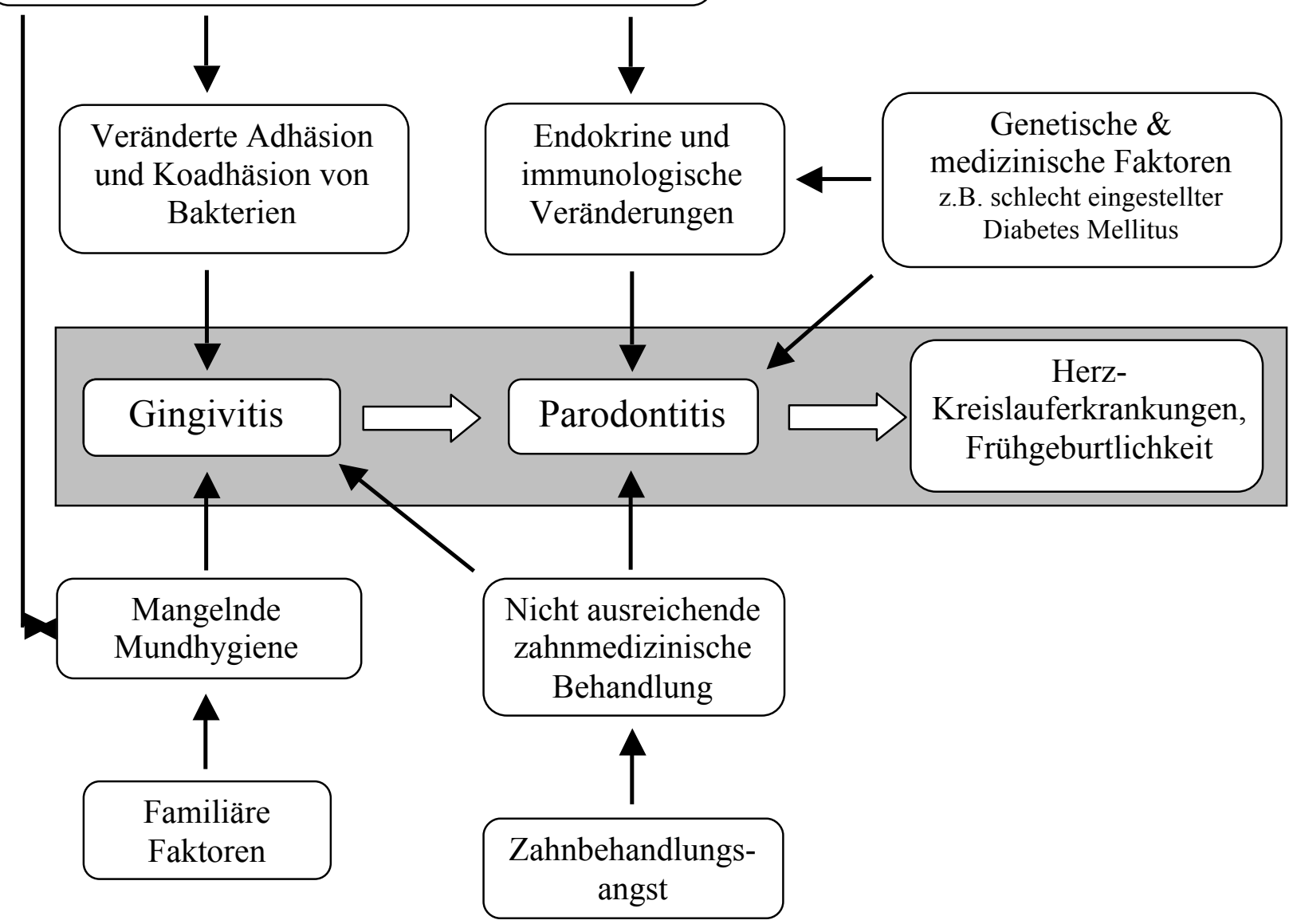

Schematische Darstellung des Zusammenspiels der wichtigsten Faktoren, die bei der individuellen Entstehung von Parodontitis von Relevanz sein können. 